\title{
Prediction of coronary heart disease mortality in Busselton, Western Australia: an evaluation of the Framingham, national health epidemiologic follow up study, and WHO ERICA risk scores
}

\author{
Matthew W Knuiman, Hien T V Vu
}

\begin{abstract}
Study objectives-To evaluate the performance of the Framingham, national health epidemiologic follow up study, and the WHO ERICA risk scores in predicting death from coronary heart disease (CHD) in an Australian population.

Design-Cohort follow up study.

Setting and participants-The cohort consisted of 1923 men and 1968 women who participated in health surveys in the town of Busselton in Western Australia over the period 1966-81. Baseline assessment included cardiovascular risk factor measurement. Mortality follow up to 31 December 1994 was used.

Main results-Risk scores for death from CHD within 10 years based on age, systolic blood pressure, cholesterol, smoking, and BMI were derived from the Busselton study data using logistic regression analysis. Similar risk scores developed from the Framingham, the national health epidemiologic follow up study, and the WHO ERICA cohorts were found to perform just as well in Busselton as the Busseltonderived scores, both before and after controlling the effect of age. There was considerable overlap across the different risk scores in the identification of individuals in the highest quintile of risk. Those in the top $20 \%$ of scores included about $41 \%$ of deaths from CHD among men and about $63 \%$ of deaths from CHD among women.

Conclusion-Although there is variation in risk score coefficients across the studies, the relative risk predictive performance of the scores is similar. The use of Framingham and other similar risk scores will not be misleading in white Australian populations.
\end{abstract}

Department of Public Health, University of Western Australia, Nedlands, Western Australia 6907, Australia

$M$ W Knuiman

H T V Vu

Correspondence to: Associate Professor M W Knuiman.

Accepted for publication January 1997

\section{(F Epidemiol Community Health 1997;51:515-519)}

Mortality from heart disease in Australia has been falling for more than 20 years, but the rates remain high and a cause for concern. ${ }^{12}$ Major well known risk factors for coronary heart disease (CHD) include raised blood pressure and cholesterol concentrations, and smoking. ${ }^{34}$ Since CHD is a multifactorial disease, risk of CHD should be estimated by assessing all cardiovascular risk factors simulta- neously. To assist clinicians with this task, early Framingham heart study investigators developed CHD risk scores for predicting the development of CHD and these have been updated in more recent publications. ${ }^{3-8}$

A number of other studies have produced risk scoring systems for men. Risk scores based on age, systolic blood pressure, cholesterol, smoking, and body mass index (BMI) have been developed from 11 studies participating in the European WHO ERICA project. Risk scores were developed separately for each of four regions and also overall. ${ }^{9}$ Data from the British regional heart study were used to develop scoring systems based on age, blood pressure, cholesterol, ECG, diabetes, current angina, and parental history of heart disease for identification of men at high risk of heart attack. The top fifth of the score distribution identified $59 \%$ of cases of ischaemic heart disease over a follow up period of five years. ${ }^{1011}$ Data from the United Kingdom heart disease prevention project were used to develop the Dundee risk score for non-fatal myocardial infarction or death from CHD over a five year period among men. ${ }^{12}$ There are few risk scores developed for women.

The generation of several risk scores has been partly motivated by the belief that risk scores developed from one population may not be applicable to another population. This is supported by a review of 15 published, estimated logistic risk functions for $\mathrm{CHD}$ morbidity/mortality which include blood pressure, cholesterol, and measures of smoking. There were noticeable differences in the coefficients and associated odds ratios between studies and the authors concluded that the "use of a scoring system derived from logistic regression to produce a measure of risk for a different population may be more misleading than advantageous". ${ }^{13}$ However, there were differences between the studies in the definition of the CHD outcome event, the length of follow up, the additional variables included in the equations, which measure of blood pressure was used (systolic or diastolic), and the definition of smoking, and these could be responsible for the differences between coefficients across studies.

A limited number of evaluations of risk scores in other settings produced findings that do not support this view. The generalisability of the Framingham CHD risk scores to other US 
populations has been examined in the "pooling project" and in a comparison with the national health epidemiologic follow up study. ${ }^{6}{ }^{14}$ These studies, which examined risk scores based on age, systolic blood pressure, cholesterol concentration, and smoking, found that the Framingham risk model was generalisable to the white population of the United States. In addition, the ERICA project found that risk scores from one region predicted mortality from CHD just as well in other regions. ${ }^{9}$ There is clearly a need for more evaluations of scoring systems.

No CHD risk scores have been developed from Australian data and none of the risk scores developed from studies in other countries have been evaluated in Australia.

The present investigation used data on CHD risk factors and mortality from the Busselton study to develop scores predicting the risk of CHD death within 10 years for men and women. The predictive performance of these scores is compared with those scores developed from the Framingham study and the national health epidemiologic follow up study (NHEFS) and the ERICA project. ${ }^{69}$

\section{Methods}

The Busselton population health studies are a series of cross sectional health surveys undertaken in the town of Busselton in the state of Western Australia. Surveys of adults were undertaken every three years from 1966-81. All persons on the electoral roll in the Shire of Busselton were invited to attend, enrolment to vote being compulsory in Australia. The participation rate in the surveys varied from $91 \%$ in 1966 to $64 \%$ in 1981 . Approximately 3500 adults attended each survey and a total of 8310 persons attended at least one survey. The conduct of the surveys, including a detailed description of the study population, the examinations of subjects, and laboratory methods have been described elsewhere. ${ }^{15-18}$ In summary, participants were asked to complete a comprehensive health and lifestyle questionnaire and to undergo various measurements and tests. Systolic and diastolic blood pressure were measured by mercury sphygmomanometer after five minutes rest in a sitting position. Serum cholesterol was determined from a blood sample and, although measured in different units over the years, all data were converted to $\mathrm{mmol} / \mathrm{l}$ for analysis. Height was measured by stadiometer for bare-footed subjects and weight was measured with subjects in light underclothes. BMI was derived as weight (in $\mathrm{kg}$ ) divided by the square of height (m). Smoking status was assessed by questionnaire. Mortality follow up from baseline survey to 1994 is currently available. Deaths are identified from linkage to the Death Register for Western Australia and through relatives. Survival status is confirmed by linkage to the electoral roll and Telecom White Pages, and through relatives. Complete follow up (to death or 1994) is available for over $99 \%$ of participants. The underlying cause of death was determined from the death certificates by a
KEY POINTS

- The usefulness of risk scoring systems for predicting death from coronary heart disease is unclear outside the populations from which they were derived.

- Risk factor and mortality data from the Busselton health study show that the Framingham, the national health epidemiologic follow up study, and the WHO ERICA risk scoring systems provide reasonable estimates in white Australian populations.

nosologist at the Australian Bureau of Statistics.

This paper focuses on risk scores based on age (years), systolic blood pressure ( $\mathrm{mm} \mathrm{Hg}$ ), cholesterol concentration ( $\mathrm{mmol} / \mathrm{l})$, current smoking (yes $/ \mathrm{no})$, and BMI $\left(\mathrm{kg} / \mathrm{m}^{2}\right)$. Analyses have been restricted to men and women 40-74 years of age at baseline. Logistic regression models for risk of death from CHD within 10 years of baseline were used to develop risk scores. Participants who died from CHD within 10 years were considered as cases and those who did not die within 10 years and those who died within 10 years from a non-CHD cause were considered non-cases. The Framingham and NHEFS risk scores evaluated in this paper were developed from logistic models for CHD death within a median follow up of 10 years for (white) men and women 40-74 years of age. ${ }^{6}$ The baseline period for the Busselton cohort was 1966-81; that for the Framingham model was 1960-64 and the period for the NHEFS model was 1971-74. The ERICA score for men aged 40-57 years was developed from a logistic model for CHD death within six years of follow up from 1982. ${ }^{9}$ Death from CHD was identified from death certificates with an underlying cause of death coded to ICD9, 410-414, in Busselton, NHEFS, and ERICA and ICD8, $410-412$ plus 414 but excluding 412.2 and 412.4, for Framingham.

The risk score was defined as the linear predictor in the logistic model. The performance of risk scores in predicting CHD death with the Busselton cohort was assessed by dividing subjects into quintiles of risk score and counting the number of observed deaths within each quintile. For the Busselton derived scores, the expected numbers of deaths in each quintile were also calculated and the goodness of fit was assessed using the Hosmer-Lemeshow test. ${ }^{19}$ In order to remove the effect of age before assessing the performance of the remaining risk factors, subjects were first divided into quintile

Table 1 Size of cohorts and number of coronary heart disease (CHD) deaths within 10 years

\begin{tabular}{lllll}
\hline & Busselton & Framingham & NHEFS & ERICA \\
\hline Men: & & & 1902 & 18931 \\
$\quad$ Total & 1923 & 1778 & 123 & 244 \\
CHD deaths & 116 & 123 & & \\
Women: & & & 2111 & - \\
$\quad$ Total & 1968 & 2235 & 58 & - \\
CHD deaths & 71 & 83 & 5 \\
\hline
\end{tabular}


Table 2 Characteristics of cohorts. Table shows mean (SD) or percentage

\begin{tabular}{lllll}
\hline & Busselton & Framingham & NHEFS & ERICA* \\
\hline Men: & & & & \\
Age (y) & $56.4(9.7)$ & $55.1(8.2)$ & $56.3(9.6)$ & 48.0 \\
Systolic BP (mmHg) & $137.1(22.2)$ & $136.7(21.6)$ & $137.0(21.1)$ & 138.4 \\
Cholesterol (mmol/l) & $6.08(1.12)$ & $6.44(1.11)$ & $5.94(1.14)$ & 6.09 \\
Smoking (\%) & 45 & 52 & 38 & 59 \\
Body mass index (kg/m $\left.{ }^{2}\right)$ & $25.6(3.3)$ & $\dagger$ & $\dagger$ & 25.8 \\
Women: & $55.8(9.3)$ & $55.7(8.3)$ & $56.1(9.6)$ & \\
Age (y) & $141.2(25.4)$ & $139.2(24.5)$ & $137.6(23.9)$ & \\
Systolic BP (mm Hg) & $6.46(1.32)$ & $6.81(1.26)$ & $6.26(1.24)$ & \\
Cholesterol (mmol/l) & 24 & 38 & 28 & \\
Smoking (\%) & $25.6(4.3)$ & $\dagger$ & $\dagger$ & \\
Body mass index $\left(\mathrm{kg} / \mathrm{m}^{2}\right)$ & & &
\end{tabular}

$\star S D$ not reported.

†Not used in Framingham and NHEFS risk scores.

groups according to age and then the risk score quintile determined within age groups.

\section{Results}

There were 1923 men and 1968 women aged 40 to 74 years and with available baseline and follow up information within the Busselton study. Over the 10 years of follow up there were 116 deaths from CHD among men and 71 among women (table 1). These cohort sizes and numbers of CHD deaths are quite similar to those for the Framingham and NHEFS studies from which risk scores were developed. The combined ERICA cohort from which the risk score for men was developed was much larger and had about twice as many deaths from CHD. ${ }^{9}$

Table 2 shows the baseline characteristics of the cohorts used to develop risk equations. The mean age at baseline was lower for ERICA since it included men aged $40-57$ years whereas the other cohorts included people aged 40 to 74 years. The cohorts are otherwise similar in all risk factors except smoking which shows some variation across studies.

As the ERICA risk score included BMI, risk scores with and without BMI were developed for Busselton. The coefficients obtained from logistic models for Busselton and those reported for the other cohorts are shown in table 3. Although the coefficients for systolic BP and smoking were considerably lower in Busselton compared with the other cohorts, none of the Busselton coefficients were significantly different (at the $p=0.05$ level) to the Framingham coefficients or the NHEFS coefficients.

The observed and expected deaths from $\mathrm{CHD}$ for each quintile of risk score are shown in table 4 . The performance of the Busselton model with age as the only risk variable is included for comparison. The HosmerLemeshow goodness of fit $p$ values indicate that the Busselton models are an adequate fit to the data. The performance of the Busselton model including BMI is essentially the same as for the model without BMI and hence is not shown. All models, including the model with only age, appear to have similar predictive performance. For each risk score, the highest quintile contained about $41 \%$ of deaths from CHD among men and about $63 \%$ of deaths from CHD among women. There were 502 men $(26 \%)$ and 482 women $(24 \%)$ who were in the highest quintile for at least one of the risk scores. This shows that there is considerable overlap across risk scores in the identification of individuals at high risk. These groups of men and women included $61(53 \%)$ and $50(70 \%)$ of the deaths from CHD among men and women respectively.

Age is clearly the dominant risk factor and it is difficult to isolate the predictive performance of the remaining risk factors in the results in table 4 . The performance of the remaining risk factors can be seen in table 5, which shows the observed and expected deaths from CHD by age group- specific risk score quintile. The results in table 4 for the model involving only age show that the risk of death from CHD with 10 years increases by a factor of more than 10 from the lowest to highest age group quintiles. After removing the effect of age as in table 5, the number of deaths from CHD increases by a factor of about 2.5 from the lowest to highest risk quintile. Thus systolic blood pressure, cholesterol, and smoking do have predictive ability after accounting for age.

The results in tables 4 and 5 both show that the Framingham, NHEFS, and ERICA risk scores perform just as well as the Busseltonderived scores despite some differences in the estimated coefficients.

\section{Discussion}

The trends in mortality and risk factors in Busselton have been similar to those in other parts of Australia, ${ }^{17}{ }^{18}$ suggesting that an assessment of the Framingham, NHEFS, and ERICA equations in Busselton is likely to provide a reasonable guide as to their usefulness in Australian populations. Our results show that

Table 3 Coefficients (SE) for risk scores developed from logistic regression models

\begin{tabular}{llllll}
\hline & $\begin{array}{l}\text { Busselton } \\
\text { (without BMI) }\end{array}$ & $\begin{array}{l}\text { Busselton } \\
\text { (with BMI) }\end{array}$ & Framingham & NHEFS & ERICA* \\
\hline Men: & $-10.57(1.07)$ & $-11.11(1.28)$ & - & - & - \\
$\quad$ Constant & $0.0835(0.0126)$ & $0.0840(0.0126)$ & $0.0784(0.0125)$ & $0.0644(0.0112)$ & 0.0996 \\
Age & $0.00933(.00426)$ & $0.00889(.00431)$ & $0.0172(.0039)$ & $0.0131(0.0041)$ & 0.0175 \\
Systolic BP & $0.219(0.084)$ & $0.213(0.084)$ & $0.148(0.084)$ & $0.175(0.080)$ & 0.258 \\
Cholesterol & - & $0.361(0.203)$ & $0.450(0.198)$ & $0.781(0.196)$ & 0.655 \\
Smoking & $-16.57(1.57)$ & $-17.43(1.77)$ & - & - & 0.0223 \\
Body mass index & $0.1604(0.0209)$ & $0.1615(0.0210)$ & $0.1081(0.0178)$ & $0.1059(0.0202)$ \\
Women: & $0.0133(0.0048)$ & $0.0126(0.0048)$ & $0.0244(0.0042)$ & $0.0223(0.0052)$ \\
$\quad$ Constant & $0.188(0.094)$ & $0.192(0.094)$ & $0.251(0.087)$ & $0.369(0.103)$ \\
Age & $0.314(0.324)$ & $0.369(0.328)$ & $0.699(0.254)$ & $0.882(0.317)$ \\
Systolic BP & - & $0.0314(0.0284)$ & - & - & \\
Cholesterol & & & & \\
Smoking & & &
\end{tabular}

$\star$ SE not reported. 
Table 4 Observed and expected coronary heart disease (CHD) deaths by quintiles of risk score and goodness of fit (GOF) $p$ values

\begin{tabular}{|c|c|c|c|c|c|c|c|}
\hline \multirow[b]{2}{*}{ Quintile } & \multicolumn{2}{|c|}{$\begin{array}{l}\text { Busselton model } \\
\text { with only age }\end{array}$} & \multicolumn{2}{|c|}{$\begin{array}{l}\text { Busselton model } \\
\text { (without BMI) }\end{array}$} & \multirow{2}{*}{$\frac{\text { Framingham }}{\text { Observed }}$} & \multirow{2}{*}{$\frac{\text { NHEFS }}{\text { Observed }}$} & \multirow{2}{*}{$\frac{E R I C A}{\text { Observed }}$} \\
\hline & Observed & Expected & Observed & Expected & & & \\
\hline \multicolumn{8}{|l|}{ Men: } \\
\hline 1 & 3 & 5.8 & 1 & 4.8 & 0 & 0 & 1 \\
\hline 2 & 10 & 10.3 & 10 & 9.5 & 9 & 8 & 7 \\
\hline 3 & 27 & 18.2 & 23 & 17.3 & 23 & 29 & 27 \\
\hline 4 & 28 & 30.6 & 35 & 30.1 & 36 & 33 & 32 \\
\hline 5 & 48 & 51.1 & 47 & 54.3 & 48 & 46 & 49 \\
\hline GOF & & $p=0.10$ & & $p=0.07$ & & & \\
\hline \multicolumn{8}{|l|}{ Women: } \\
\hline 1 & 0 & 0.6 & 0 & 0.4 & 0 & 0 & \\
\hline 2 & 0 & 1.8 & 0 & 1.6 & 1 & 2 & \\
\hline 3 & 6 & 5.1 & 5 & 4.9 & 3 & 3 & \\
\hline 4 & 20 & 14.7 & 19 & 14.0 & 22 & 24 & \\
\hline & 45 & 48.8 & 47 & 51.9 & 45 & 42 & \\
\hline GOF & & $p=0.18$ & & $\mathrm{p}=0.22$ & & & \\
\hline
\end{tabular}

the Framingham, NHEFS, and ERICA risk scores perform just as well in Busselton as Busselton-derived scores. This is in accord with previous evaluations of Framingham scores in other populations. ${ }^{614}$ When the development of a risk score and the evaluation of its performance are based on the same data, the performance may be over-estimated. Such bias may be avoided through cross-validation. The performance of the Busselton-derived scores did not noticeably diminish when crossvalidation was used.

As age is not a modifiable risk factor it is desirable to assess separately the predictive performance of the modifiable risk factors systolic blood pressure, cholesterol, and smoking. Some evaluation studies have fixed the value of age in the risk score to be a constant for all individuals. ${ }^{6}{ }^{12}$ However, this technique does not allow the comparison of observed and expected cases and, since many risk factors are positively correlated with age, the mean age still increases across risk score groups and hence the effect of age is only partly eliminated. In this paper we have eliminated the effect of age by first dividing individuals into age groups and then calculating risk score quintiles separately within age groups.

The risk scores considered here have been derived from logistic regression models. These methods require a fixed follow up period (eg 10 years) and the outcome is reduced to whether or not the subject died from CHD during the period of follow up. A death at two years and a death at eight years are treated as being the same. When follow up is available for variable

Table 5 Observed and expected coronary heart disease (CHD) deaths in the cohorts by age specific quintiles of risk score

\begin{tabular}{|c|c|c|c|c|c|}
\hline \multirow[b]{2}{*}{ Quintile } & \multicolumn{2}{|c|}{ Busselton (without BMI) } & \multirow{2}{*}{$\begin{array}{l}\text { Framingham } \\
\text { Observed }\end{array}$} & \multirow{2}{*}{$\frac{\text { NHEFS }}{\text { Observed }}$} & \multirow{2}{*}{$\frac{\text { ERICA }}{\text { Observed }}$} \\
\hline & Observed & Expected & & & \\
\hline \multicolumn{6}{|l|}{ Men: } \\
\hline 1 & 15 & 15.0 & 15 & 13 & 13 \\
\hline 2 & 14 & 18.3 & 16 & 19 & 18 \\
\hline 3 & 24 & 21.6 & 22 & 26 & 21 \\
\hline 4 & 28 & 26.0 & 25 & 25 & 30 \\
\hline 5 & 35 & 35.4 & 38 & 33 & 34 \\
\hline \multicolumn{6}{|l|}{ Women: } \\
\hline 1 & 9 & 7.7 & 8 & 8 & \\
\hline 2 & 9 & 11.0 & 11 & 10 & \\
\hline 3 & 16 & 12.9 & 16 & 17 & \\
\hline 4 & 13 & 16.5 & 10 & 12 & \\
\hline 5 & 24 & 24.6 & 26 & 24 & \\
\hline
\end{tabular}

lengths of time for subjects this method does not allow full use of the available information. The Cox proportional hazards model uses the individual survival times thus not wasting information and allowing the estimation of probabilities for a range of follow up periods. ${ }^{20}$ Also, in logistic regression, all deaths from non-CHD causes within the fixed follow up period are treated as non-cases regardless of whether they occurred early or late in the period. In Cox regression such participants are deemed not to have died from CHD up to the time of the non-CHD death. This difference could lead to under-estimation of coefficients in logistic as compared to Cox regression. Leaverton et al reported logistic regression coefficients for mortality from CHD within a median follow up period of 10 years for Framingham and the NHEFS cohorts. ${ }^{6}$ The length of follow up varied however between 9 and 11 years for Framingham and between 7 and 13 years for NHEFS. They also reported that coefficients from the Cox proportional hazards model were very nearly identical to those from logistic regression. When we fitted Cox models to the Busselton cohort with mortality follow up to the end of 1994 (median follow up 19 years), the coefficients were similar to those from the logistic models except that the coefficient for smoking increased to about 0.4. Differences between the logistic and Cox models may be more apparent when periods of follow up have greater variation.

The coefficients of the risk scores compared and evaluated in this paper were not very precisely estimated. This was due to the small number of deaths from CHD in each of the cohorts. The MRFIT study involved 316099 men aged 35-57 years and 6,327 died from CHD over an average period of follow up of 12 years. Although a risk score has not been explicitly developed from these data, the coefficients from the Cox proportional hazards model were quite similar to those of the risk scores evaluated here. ${ }^{4}$ The Dundee coronary risk score and a multiple logistic equation estimated from a large cohort of Italian men also had similar coefficients. ${ }^{12} 21$

In this paper we have evaluated the performance of several scoring systems in separating low risk from high risk individuals, that is, in assessing relative risk. We have not evaluated their performance in assessing absolute risk. The constant terms in the logistic equations are needed for this assessment and they were not published. Others have found that scoring systems that assess relative risk well in other populations do not necessarily assess absolute risk well when the incidence of CHD is much lower or higher. ${ }^{22} 23$

There are many known risk factors for death from CHD in addition to blood pressure, cholesterol, smoking, and BMI. They include lipid fractions, angina, ECG abnormalities, diabetes, left ventricular hypertrophy, family history of heart disease, and more relevant measures of obesity such as waist/hip ratio. Also, there is now more intense treatment of risk factors. Where practical, these risk factors and any 
current treatments should also be incorporated into risk scoring systems.

CHD risk scores allow a quantitative multivariate assessment of risk and are useful in clinical and general practice settings for identification of individuals at high risk and for monitoring changes in risk. ${ }^{12} 124$ Simply counting up the number of highly elevated risk factors is inadequate as it fails to identify individuals who have several moderately elevated risk factors but no highly elevated risk factors. ${ }^{25}$

Another use of quantitative risk scores is in the evaluation of community interventions. Studies assessing the effect of community-wide interventions on CHD generally have too little power or follow up to use deaths from CHD as an endpoint. Consequently multivariate risk scores have been used to test for overall differences in CHD risk between intervention and control communities. ${ }^{26-28}$

In conclusion, this investigation has shown that although there is variation in risk score coefficients across studies, the relative risk predictive performance of the scores is similar. The use of Framingham and other similar risk scores will not be misleading in white Australian populations.

The authors acknowledge the programming work of Ms Helen Bartholomew in preparing the data files.

Funding: this project was supported by the Western Australian Health Promotion Foundation.

Conflicts of interest: none.

1 Al-Roomi KA, Dobson AJ, Hall E, Heller RF, Magnus P. Declining mortality from ischaemic heart disease and cerebrovascular disease in Australia. $\mathrm{Am} \mathcal{J}$ Epidemiol 1989;129:503-10.

2 Thompson PL, Hobbs MST, Martin CA. The rise and fall of ischaemic heart disease in Australia. Aust NZ 7 Med 1988;18:327-37.

3 Kannel WB, McGee D, Gordon T. A general cardiovascular risk profile: the Framingham study. Am $\mathcal{f}$ Cardiol $1976 ; 38$ $46-51$.

4 Neaton JD, Wentworth D. Serum cholesterol, blood pressure, cigarette smoking, and death from coronary heart disease. Arch Intern Med 1992;152:56-64.

5 Truett J, Cornfield J, Kannel W. Multivariate analysis of the risk of coronary heart disease in Framingham. $\mathcal{f}$ Chron Dis 1967;20:511-24.

6 Leaverton PE, Sorlie PD, Kleinman JC, et al. Representativeness of the Framingham risk model for coronary heart disease mortality: a comparison with a national cohort disease mortality: a comparison with
study. F Chron Dis 1987;40:775-84.

7 Levy D, Wilson PWF, Anderson KM, Castelli WP. Stratifying the patient at risk from coronary heart disease: new insights from the Framingham heart study. Am Heart $\mathcal{f}$ 1990;119:712-7.
8 Anderson KM, Wilson PWF, Odell PM, Kannel WB. An updated coronary risk profile: a statement for health professionals. Circulation 1991;83:356-62.

9 ERICA Research Group. Prediction of coronary heart disease in Europe. The 2 nd report of the WHO-ERICA project. Eur Heart f 1991;12:291-7.

10 Shaper AG, Pocock SJ, Phillips AN, Walker M. Identifying men at high risk of heart attacks: strategy for use in general practice. BMF 1986;293:474-9.

11 Shaper AG, Pocock SJ, Phillips AN, Walker M. A scoring system to identify men at high risk of a heart attack. Health Trends 1987;19:37-9.

12 Tunstall-Pedoe $H$. The Dundee coronary risk-disk for management of change in risk factors. BMF 1991;303:744-47.

13 Chambless LE, Dobson AJ, Patterson CC, Raines B. On the use of a logistic risk score in predicting risk of coronary heart disease. Stat Med 1990;9:385-96.

$14 \mathrm{McGee} D$, Gordon T. The results of the Framingham study applied to four other US-based epidemiologic studies of cardiovascular disease. In: Kannel WB, Gordon T eds. The Framingham study: An epidemiologic investigation of cardioFramingham study: An epidemiologic investigation of cardio
vascular disease. DHEW Publication No NIH 76-1083. Washington DC, US Government Printing Office, 1976.

15 Cullen KJ. Mass health examinations in the Busselton population, 1966 to 1970 . Med F Aust 1972;2:714-8.

16 Webster IW. Busselton, a community study in cardiovascular disease. Aust NZ F Med 1974;4:412-4.

17 Knuiman MW, Cullen KJ, Bulsara MK, Welborn TA Hobbs MST. Mortality trends, 1965 to 1989 , in Busselton, the site of repeated health surveys and interventions. Aust $\mathcal{F}$ Public Health 1994;18:129-35.

18 Knuiman MW, Jamrozik K, Welborn TA, Bulsara MK, Divitini ML, Whittall DE. Age and secular trends in risk factors for cardiovascular disease in Busselton. Aust 7 Public Health 1995;19:375-82.

19 Hosmer DW, Lemeshow S. Applied logistic regression. New York: John Wiley, 1989.

20 Kalbfleisch JD, Prentice RL. The statistical analysis of failure time data. New York: John Wiley, 1980.

21 Menotti A, Farchi G, Seccareccia F. The prediction of coronary heart disease mortality as a function of major risk factors in over 30,000 men in the Italian RIFLE pooling project. A comparison with the MRFIT primary screenees. The RIFLE research group. $f$ Cardiovascular Risk 1994;1: 263-70.

22 Keys A, Menotti A, Aravanis C, et al. The seven countries study: 2,289 deaths in 15 years. Prev Med 1984;13:141-54

23 Menotti A, Keys A, Kromhout D, et al. Twenty-five-year mortality from coronary heart disease and its prediction in five cohorts of middle-aged men in Finland, The Netherlands, and Italy. Prev Med 1990;19:270-8.

24 Thorsen RD, Jacobs D, Grimm RH, Keys A, Taylor $H$, Blackburn H. Preventive cardiology in practice: a device for risk estimation and counselling in coronary disease. Prev Med 1979;8:548-56.

25 Kannel WB, McGee DL. Composite scoring - methods and predictive validity: insights from the Framingham Study. Health Services Res 1987;22:501-35.

26 Williams PT, Fortmann SP, Farquhar JW, Varady A, Mellen S. A comparison of statistical methods for evaluating risk factor changes in community-based studies: an example from the Stanford three-community study. $f$ Chronic Dis from the Stanford

27 Farquhar JW, Fortmann SP, Flora JA, et al. Effects of community-wide education on cardiovascular disease risk factors. The Stanford five-city project. $\mathcal{F} A M A$ 1990;264: 359-65.

28 Luepker RV, Murray DM, Jacobs DR, et al. Community education for cardiovascular disease prevention: risk factor changes in the Minnesota heart health program. Am $f \mathrm{Pub}$ lic Health 1994;84:1383-93. 